\title{
Research Article \\ Bohm Quantum Trajectories of Scalar Field in Trans-Planckian Physics
}

\author{
Jung-Jeng Huang \\ Physics Division, Department of Mechanical Engineering, Ming Chi University of Technology, Taishan, \\ New Taipei City 24301, Taiwan \\ Correspondence should be addressed to Jung-Jeng Huang, huangjj@mail.mcut.edu.tw
}

Received 17 October 2011; Revised 7 December 2011; Accepted 29 December 2011

Academic Editor: Kadayam S. Viswanathan

Copyright (C) 2012 Jung-Jeng Huang. This is an open access article distributed under the Creative Commons Attribution License, which permits unrestricted use, distribution, and reproduction in any medium, provided the original work is properly cited.

\begin{abstract}
In lattice Schrödinger picture, we investigate the possible effects of trans-Planckian physics on the quantum trajectories of scalar field in de Sitter space within the framework of the pilot-wave theory of de Broglie and Bohm. For the massless minimally coupled scalar field and the Corley-Jacobson type dispersion relation with sextic correction to the standard-squared linear relation, we obtain the time evolution of vacuum state of the scalar field during slow-roll inflation. We find that there exists a transition in the evolution of the quantum trajectory from well before horizon exit to well after horizon exit, which provides a possible mechanism to solve the riddle of the smallness of the cosmological constant.
\end{abstract}

\section{Introduction}

The scenario of inflationary cosmology successfully provides the paradigm for generating the inhomogeneities which seed the structures of the universe we observe today [1]. In the simplest inflationary model, these inhomogeneities arise from the quantum fluctuations in a single scalar field about its vacuum state. The conventional choice of a vacuum during inflation is the Bunch-Davies (BD) vacuum [2]. However, it is well known that the notion of a vacuum state during inflation is ambiguous in quantum theory [3], and the choice of initial quantum vacuum state affects the predictions of inflation $[4,5]$.

Recently Perez et al. [6] discussed how predictions for the cosmic microwave background $(\mathrm{CMB})$ could be affected by a hypothetical dynamical collapse of the wave function. As a different possibility, the notion of quantum nonequilibrium $[7,8]$ was also discussed in terms of the pilot-wave theory of de Broglie and Bohm [9-13] and was later generalized to include all deterministic hidden-variables theories [14]. In the context of inflationary cosmology, a deterministic hidden-variables theory allows the existence of 
vacuum states with nonstandard or nonequilibrium field fluctuations, resulting in statistical predictions that deviate from those of quantum theory $[15,16]$.

Moreover, the inflationary scenario has a serious trans-Planckian problem, which is whether the predictions of standard cosmology are insensitive to effects of the transPlanckian physics. Since there is no successful quantum gravity theory to handle the physics around the Planck scale, one of the methods is to use the effective nonlinear dispersion relations to mimic the effects of the trans-Planckian physics. For example, the CorleyJacobson (CJ) type dispersion relations were used to study the possible effects of the trans-Planckian physics on cosmological perturbations [17-19]. Note also that the CJ type dispersion relations can also be derived naturally from the recently proposed quantum gravity model called Horava-Lifshitz (HL) gravity [20-22].

In our previous papers [23-26], we used the lattice Schrödinger picture to study the free scalar field theory in de Sitter space, derived the wave functionals for the BD vacuum state and its excited states, and found the corresponding de Broglie and Bohm quantum trajectories. The purpose of this paper is to study further the possible effects of the transPlanckian physics on the quantum trajectories of scalar field in de Sitter space within the framework of the pilot-wave theory of de Broglie and Bohm. Throughout this paper, we will set $\hbar=c=1$.

\section{Pilot-Wave Scalar Field in De Sitter Space}

We consider the scalar field theory which has the Lagrangian density

$$
\begin{gathered}
L=|g|^{1 / 2}\left\{\frac{1}{2}\left[g^{\mu v}(x) \phi(x)_{, \mu} \phi(x)_{, \nu}\right]-V(\phi)\right\}, \\
V(\phi)=\frac{m^{2} \phi^{2}}{2}+\frac{\xi R \phi^{2}}{2},
\end{gathered}
$$

where $\phi$ is a real scalar field, $V(\phi)$ is the potential, $m$ is the mass of the scalar quanta, $R$ is the Ricci scalar curvature, $\xi$ is the coupling parameter, and $g=\operatorname{det} g_{\mu \nu}, \mu, v=0,1, \ldots, d$. For a spatially flat $(1+d)$-dimensional Robertson-Walker spacetime with scale factor $a(t)$, we have

$$
\begin{aligned}
d s^{2} & =d t^{2}-a^{2}(t) d^{2} x^{i}, \quad i=1,2, \ldots, d, \\
L & =a^{d}\left\{\frac{1}{2}\left[\left(\partial_{0} \phi\right)^{2}-a^{-2}\left(\partial_{i} \phi\right)^{2}\right]-V(\phi)\right\} .
\end{aligned}
$$

In the $(1+d)$-dimensional de Sitter space, we have $a(t)=\exp (h t)$, where $h \equiv \dot{a} / a$ is the Hubble parameter which is a constant. Note that in three spatial dimensions $d=3$, the curvature $R=12 h^{2}$ is also a constant. Throughout this paper, we use this exact de Sitter spacetime background to describe the inflationary era, which is only a special case ( $n \rightarrow \infty$ limit) of power-law inflation with $a(t)=a_{0} t^{n}$. For mathematical simplicity, we consider the case of $d=1$ in the following. The extention to higher spatial dimension is straightforward without changing the nature of our results. 
In the lattice formalism, we have the following changes:

$$
\begin{aligned}
\phi(x, t) & \longrightarrow \phi_{j}(t), \\
\partial_{0} \phi(x, t) & \longrightarrow \dot{\phi}_{j}, \quad j=1,2, \ldots, N, \\
\partial_{1} \phi(x, t) & \longrightarrow \frac{\left|\phi_{j}(t)-\phi_{j-1}(t)\right|}{\varepsilon},
\end{aligned}
$$

and the Lagrangian reads

$$
L=\sum_{j=1}^{N}\left\{\frac{1}{2}\left[\dot{\tilde{\phi}}_{j}-\frac{1}{2} h \tilde{\phi}_{j}\right]^{2}-\frac{1}{2} \frac{\left(\tilde{\phi}_{j}-\tilde{\phi}_{j-1}\right)^{2}}{a^{2} \varepsilon^{2}}-\frac{1}{2}\left(m^{2}+\xi R\right) \tilde{\phi}_{j}^{2}\right\}
$$

where $\tilde{\phi}_{j} \equiv a^{1 / 2} \varepsilon^{1 / 2} \phi_{j}$, and $\varepsilon=W / N$; that is, $W$ is the overall comoving spatial size of lattice. From (2.4) we obtain the Hamiltonian

$$
H=\sum_{j=1}^{N}\left\{\frac{1}{2} \tilde{p}_{j}^{2}+\frac{1}{2} h \tilde{p}_{j} \tilde{\phi}_{j}+\frac{1}{2} \frac{\left(\tilde{\phi}_{j}-\tilde{\phi}_{j-1}\right)^{2}}{a^{2} \varepsilon^{2}}+\frac{1}{2}\left(m^{2}+\xi R\right) \tilde{\phi}_{j}^{2}\right\}
$$

where $\tilde{p}_{j}$ is the conjugate momentum of $\tilde{\phi}_{j}$,

$$
\tilde{p}_{j}=\frac{\partial}{\partial \dot{\tilde{\phi}}_{j}} \mathrm{~L}=\dot{\tilde{\phi}}_{j}-\frac{1}{2} h \tilde{\phi}_{j}
$$

Then we consider the discrete Fourier transforms

$$
\begin{array}{rlrl}
\tilde{\phi}_{j} & =\sum_{l=1}^{N} \frac{\exp [-i j l 2 \pi / N]}{\sqrt{N}} \phi_{l}, & & \phi_{l}=\sum_{j=1}^{N} \frac{\exp [i j l 2 \pi / N]}{\sqrt{N}} \tilde{\phi}_{j}, \\
\tilde{p}_{j}=\sum_{l=1}^{N} \frac{\exp [i j l 2 \pi / N]}{\sqrt{N}} p_{l}, & p_{l}=\sum_{j=1}^{N} \frac{\exp [-i j l 2 \pi / N]}{\sqrt{N}} \tilde{p}_{j},
\end{array}
$$

such that

$$
\begin{gathered}
\left\{\tilde{\phi}_{j}, \tilde{p}_{j}\right\}=\left\{\tilde{\phi}_{j+N}, \tilde{p}_{j+N}\right\}, \quad\left\{\phi_{l}, p_{l}\right\}=\left\{\phi_{l+N}, p_{l+N}\right\}, \\
\left\{\phi_{N-l}, p_{N-l}\right\}=\left\{\phi_{-l}, p_{-l}\right\}=\left\{\phi_{l}{ }^{*}, p_{l}{ }^{*}\right\} .
\end{gathered}
$$


Here $\left(\phi_{l}, p_{l} \mid l=1,2, \ldots, N / 2\right)$ can be chosen as the independent variables which obey the Poisson brackets $\left\{\phi_{l}, p_{l}\right\}=\left\{\tilde{\phi}_{j}, \tilde{p}_{j}\right\}$. From (2.7) we can obtain the following identities:

$$
\begin{array}{ll}
\sum_{j=1}^{N} \tilde{p}_{j}^{2}=\sum_{l=1}^{N} p_{l}{ }^{*} p_{l}, & \sum_{j=1}^{N} \tilde{p}_{j}^{2} \tilde{\phi}_{j}=\sum_{l=1}^{N} p_{l} \phi_{l}, \\
\sum_{j=1}^{N} \tilde{\phi}_{j}^{2}=\sum_{l=1}^{N} \phi_{l}{ }^{*} \phi_{l}, & \sum_{j=1}^{N}\left(\tilde{\phi}_{j}-\tilde{\phi}_{j-1}\right)^{2}=\sum_{l=1}^{N} 4 \sin ^{2}\left(\frac{l \pi}{N}\right) \phi_{l}^{*} \phi_{l} .
\end{array}
$$

Furthermore, we define

$$
\begin{gathered}
\omega_{l} \equiv \frac{2}{\varepsilon} \sin \left(\frac{l \pi}{N}\right), \\
\phi_{l}=\phi_{1 l}+i \phi_{2 l}, \quad p_{l}=p_{1 l}+i p_{2 l},
\end{gathered}
$$

where $p_{l}$ is the conjugate momentum for $\phi_{l}$, and the subscripts 1 and 2 denote the real and imaginary parts, respectively. Therefore, the Hamiltonian (2.5) becomes in momentum space

$$
\begin{gathered}
H=2 \sum_{l=1}^{N / 2} \sum_{r=1}^{2} H_{r l} \\
H_{r l}=\frac{1}{2} p_{r l}{ }^{2}+\frac{1}{2} h p_{r l} \phi_{r l}+\frac{1}{2} a^{-2} \omega_{l}^{2} \phi_{r l}{ }^{2}+\frac{1}{2}\left(m^{2}+\xi R\right) \phi_{r l}{ }^{2} .
\end{gathered}
$$

To quantize the theory above, we note also that each pair of operators $\hat{\phi_{r l}}$ and $\hat{p_{r l}}$ must satisfy the equal time commutation relations $\left[\hat{\phi_{r l}}, \hat{p_{r l}}\right]=i$. These commutation relations are realized by the representation

$$
\phi_{r l} \longrightarrow \phi_{r l}, \quad p_{r l} \longrightarrow-\frac{i \partial}{\partial \phi_{r l}}
$$

that is, the functional Schrödinger representation, which is not so widely used as the Fock representation. These operators act on wave functional $\psi(\phi)$, and the inner product is given by $\left\langle\psi_{1} \mid \psi_{2}\right\rangle=\int\left(\prod_{r l} D \phi_{r l}\right) \psi_{1}{ }^{*}(\phi) \psi_{2}(\phi)$. With the field basis $|\phi\rangle$, that is, $|\phi\rangle$ are the eigenstates of the operator $\hat{\phi}_{r l}, \hat{\phi}_{r l}|\phi\rangle=\phi_{r l}|\phi\rangle$, and then the wave functional $\psi(\phi, t)$ can be regarded as the expansion coefficient $\langle\phi \mid \psi(t)\rangle$ of the state $|\psi(t)\rangle$. The dynamics of the wave functional is, therefore, determined by the functional Schrödinger equation

$$
H \psi=i \frac{\partial}{\partial t} \psi,
$$

with $H$ defined by (2.11). Writing $\psi$ as the product form

$$
\psi\left[\phi_{r l}, t\right]=\prod_{l=1}^{N / 2} \prod_{r=1}^{2} \psi_{r l}\left(\phi_{r l}, t\right) \equiv \prod_{r l} \psi_{r l}\left(\phi_{r l}, t\right),
$$


we obtain from (2.13) the following:

$$
H_{r l} \psi_{r l}=i \frac{\partial}{\partial t} \psi_{r l}, \quad r=1,2
$$

Rewriting (2.15) as the following form:

$$
\left\{\frac{1}{2}\left(p_{r l}+\frac{1}{2} h \phi_{r l}\right)^{2}-\frac{1}{8} h^{2} \phi_{r l}{ }^{2}+\frac{1}{2} \alpha^{-2} \omega_{l}^{2} \phi_{r l}{ }^{2}+\frac{1}{2}\left(m^{2}+\xi R\right) \phi_{r l}{ }^{2}\right\} \psi_{r l}=i \frac{\partial}{\partial t} \psi_{r l},
$$

and setting $P_{r l}=p_{r l}+1 / 2 h \phi_{r l}=-i \partial / \partial \phi_{r l}$, so that $\left[\phi_{r l}, P_{r l}\right]=\left[\phi_{r l}, p_{r l}\right]=i$, gives the timedependent Schrödinger equation

$$
-\frac{1}{2} \frac{\partial^{2} \psi_{r l}}{\partial \phi_{r l}{ }^{2}}+\frac{1}{2}\left[a^{-2} \omega_{l}^{2}+\left(m^{2}+\xi R\right)-\frac{1}{4} h^{2}\right] \phi_{r l}^{2} \psi_{r l}=i \frac{\partial \psi_{r l}}{\partial t}
$$

To solve (2.17), we use the conformal time $\tau$ defined by $d \tau=d t / a$

$$
\tau=-h^{-1} \exp (-h t)=-h^{-1} a^{-1}, \quad-\infty<\tau<0 .
$$

Then, for each real mode $\phi_{r l}$, the normalized instantaneous vacuum and its excited states are found to be [24]

$$
\begin{aligned}
\psi_{\left(n_{r l}\right)}\left(\phi_{r l}, \tau\right) & =A_{l\left(n_{r l}\right)}(\tau) H_{\left(n_{r l}\right)}\left(\eta_{r l}(\tau)\right) \exp \left(-\frac{1}{2} B_{l}(\tau) a^{-1} \phi_{r l}{ }^{2}\right), \\
n_{r l} & =0,1,2, \ldots, \quad \eta_{r l}(\tau) \equiv g(\tau) \omega_{l}^{1 / 2} a^{-1 / 2} \phi_{r l} .
\end{aligned}
$$

Here $H_{\left(n_{r l}\right)}$ is the $n$ th-order Hermite polynomial, and the complex function $B_{l}(\tau)$, real function $g(\tau)$, and complex function $A_{l\left(n_{r l}\right)}(\tau)$ are given by (2.20), (2.21), and (2.22), respectively

$$
\begin{gathered}
B_{l}(\tau)=\frac{2 / \pi|\tau|}{\left|H_{v}{ }^{(1)}\right|^{2}}-i \frac{\omega_{l}}{2} \frac{\left(\left|H_{v}{ }^{(1)}\right|^{2}\right)^{\prime}}{\left|H_{v}{ }^{(1)}\right|^{2}}, \\
g(\tau)=\left(\frac{2}{\pi \omega_{l}|\tau|}\right)^{1 / 2} \frac{1}{\left|H_{v}{ }^{(1)}\right|}, \\
A_{l\left(n_{r l}\right)}(\tau)=\exp \left[-i \frac{1}{2} \int B_{l}(\tau) d \tau-i n_{r l} \omega_{l} \int g^{2}(\tau) d \tau+\text { const }\right],
\end{gathered}
$$


where $H_{v}{ }^{(1)}\left(\omega_{l}|\tau|\right)$ is the Hankel function of the first kind and of order $v$, with $v^{2}=1 / 4-$ $\left(m^{2}+\xi R\right) / h^{2}$, and the prime in (2.20) denotes the derivative with respect to $\omega_{l}|\tau|$. Note that the wave functionals for each real mode $\phi_{r l}$ can also be rewritten in the following form [25]:

$$
\psi_{r l\left(n_{r l}\right)}\left(\phi_{r l}, \tau\right)=R_{\left(n_{r l}\right)}\left(\phi_{r l}, \tau\right) \exp \left[i \Theta_{\left(n_{r l}\right)}\left(\phi_{r l}, \tau\right)\right], \quad n_{r l}=0,1,2, \ldots,
$$

with the amplitude

$$
\begin{gathered}
R_{\left(n_{r l}\right)}\left(\phi_{r l}, \tau\right)=\left[\frac{\sqrt{2 h / \pi}}{\sqrt{\pi} 2^{n} n_{r l} !\left|H_{v}^{(1)}\right|}\right]^{1 / 2} H_{\left(n_{r l}\right)}\left(\eta_{r l}\right) \exp \left(-\frac{1}{2} \eta_{r l}{ }^{2}\right), \\
\eta_{r l}=\frac{\sqrt{2 h / \pi}}{\left|H_{v}{ }^{(1)}\right|} \phi_{r l},
\end{gathered}
$$

and the phase

$$
\Theta_{\left(n_{r l}\right)}\left(\phi_{r l}, \tau\right)=-\frac{h \omega_{l}|\tau|}{2} \frac{\left(\left|H_{\nu}{ }^{(1)}\right|\right)^{\prime}}{\left|H_{v}{ }^{(1)}\right|} \phi_{r l}{ }^{2}-\left(\frac{1}{2}+n_{r l}\right) \int \frac{2 / \pi|\tau|}{\left|H_{\nu}{ }^{(1)}\right|^{2}} d \tau .
$$

The complete state wave functionals can be written as $\psi_{[n]}\left[\phi_{r l}, t\right]=\prod_{r l} \psi_{\left(n_{r l}\right)}\left(\phi_{r l}, t\right)$, where $n_{r l}=0,1,2, \ldots$ and $[n] \equiv\left(n_{i}, n_{j}, \ldots\right)$ which means that it is possible for different field modes to be in different excited states; that is, mode $i$ is in the $n_{i}$-excited state, mode $j$ is in the $n_{j}$ excited state, and so forth. For $n_{r l}=0$, the ground state wave functional corresponds to the standard $\mathrm{BD}$ vacuum chosen conventionally in the literature. For the case of spatial dimension $d=3$, we have $v^{2}=9 / 4-\left(m^{2}+\xi R\right) / h^{2}$ with $R=12 h^{2}$, and the mode index $l$ in $\omega_{l}$ carries labels $\left(l_{i}, i=1,2,3\right)$ which will be suppressed below.

To define the pilot-wave scalar field theory, we note from (2.13)-(2.17) that, in the case of $d=3$, the time-dependent Schrödinger equation for $\psi$ is given by

$$
i \frac{\partial \psi}{\partial t}=\sum_{r l}\left\{-\frac{1}{2} \frac{\partial^{2}}{\partial \phi_{r l}^{2}}+\frac{1}{2}\left[a^{-2} \omega_{l}^{2}+\left(m^{2}+\xi R\right)-\frac{9}{4} h^{2}\right] \phi_{r l}^{2}\right\} \psi
$$

which implies the continuity equation

$$
\frac{\partial|\psi|^{2}}{\partial t}+\sum_{r l}\left\{\frac{\partial}{\partial \phi_{r l}}\left[|\psi|^{2} \frac{\partial \Theta}{\partial \phi_{r l}}\right]\right\}=0
$$

and the de Broglie velocities

$$
\frac{d \phi_{r l}}{d t}=\frac{\partial \Theta}{\partial \phi_{r l}}
$$


where $\psi=|\psi| \exp [i \Theta]$. The wave functional $\psi_{r l}\left(\right.$ where $\psi_{r l}=\left|\psi_{r l}\right| \exp \left[i \Theta_{r l}\right]$, and $\left.\Theta=\sum_{r l} \Theta_{r l}\right)$ for a single mode $\phi_{r l}$ satisfies (2.17), which implies the continuity equation

$$
\frac{\partial\left|\psi_{r l}\right|^{2}}{\partial t}+\frac{\partial}{\partial \phi_{r l}}\left(\left|\psi_{r l}\right|^{2} \frac{\partial \Theta_{r l}}{\partial \phi_{r l}}\right)=0
$$

and the de Broglie velocity field

$$
\frac{d \phi_{r l}}{d t}=\frac{\partial \Theta_{r l}}{\partial \phi_{r l}}
$$

Here $\psi$ is interpreted as a physical field in field configuration space, guiding the evolution of $\phi_{r l}$.

Since the exact forms of the normalized instantaneous BD vacuum and its excited states are given by (2.23), it is straightforward to find the corresponding quantum trajectories of these eigenstates by solving (2.30) for each mode. Substituting the phase (2.25) into (2.30) and using the conformal time $\tau$ yields the de Broglie velocity field for $\phi_{r l}$

$$
\frac{d \phi_{r l}}{d \tau}=a \frac{\partial \Theta_{(n)}\left(\phi_{r l}, \tau\right)}{\partial \phi_{r l}}=-\omega_{l} \frac{\left(\left|H_{\nu}{ }^{(1)}\right|\right)^{\prime}}{\left|H_{v}{ }^{(1)}\right|} \phi_{r l}
$$

In the continuum limit $\left(\omega_{l} \rightarrow k\right),(2.31)$ reads

$$
\frac{d \phi_{r k}}{d \tau}=-k \frac{\left(\left|H_{v}{ }^{(1)}(k|\tau|)\right|\right)^{\prime}}{\left|H_{v}{ }^{(1)}(k|\tau|)\right|} \phi_{r k}
$$

which has the solution

$$
\phi_{r k}(z)=C\left|H_{v}^{(1)}(z)\right|
$$

where $z \equiv k|\tau|=k / a / h$ is the ratio of physical wave number to the inverse of Hubble radius, and integration constant $C$ is chosen to be $\phi_{r k}\left(z_{0}\right) /\left|H_{v}{ }^{(1)}\left(z_{0}\right)\right|$ with $z_{0}$ being some reference point. Note that the quantum trajectory (2.33) is independent of the quantum number $n_{r l}$ and depends on the form of the potential $V(\phi)$ through $v$ [26]. 


\section{Evolution of Vacuum Wave Functional in the Trans-Planckian Physics}

To investigate the effect of the trans-Planckian physics, we consider the Corley-Jacobson type dispersion relations

$$
\omega^{2}\left(\frac{k}{a}\right)=k^{2}\left[1+b_{s}\left(\frac{k}{a M}\right)^{2 s}\right]
$$

where $M$ is a cut-off scale, $s$ is an integer, and $b_{s}$ is an arbitrary coefficient [17-19]. Note that the action for a scalar field with the modified dispersion relation (3.1) with $s=1$ and $b_{1}>0$ takes the form $[27,28]$

$$
S=\int d^{4} x \sqrt{-g}\left(L_{\phi}+L_{\mathrm{cor}}+L_{u}\right)
$$

where $L_{\phi}$ is the standard Lagrangian of a minimally coupled scalar field

$$
L_{\phi}=\frac{1}{2} g^{\mu \nu} \partial_{\mu} \phi \partial_{\nu} \phi-V(\phi)
$$

$L_{\text {cor }}$ corresponds to the nonlinear part of the dispersion relation

$$
L_{\mathrm{cor}}=-\frac{b_{1}}{M^{2}}\left(D^{2} \phi\right)^{2}
$$

and $L_{u}$ describes the dynamics of a unit time-like vector field $u^{\mu}$ which defines a preferred rest frame

$$
L_{u}=-\lambda\left(g^{\mu v} u_{\mu} u_{v}-1\right)-d_{1} F^{\mu v} F_{\mu \nu}
$$

with

$$
\begin{aligned}
D^{2} \phi & =\perp^{\alpha \beta} \nabla_{\alpha} \nabla_{\beta} \phi+u^{\alpha} \nabla_{\alpha} \phi \nabla_{\beta} u^{\beta}, \\
\perp_{\mu \nu} & \equiv-g_{\mu \nu}+u_{\mu} u_{v}, \\
F_{\mu \nu} & \equiv \nabla_{\mu} u_{v}-\nabla_{\nu} u_{\mu} .
\end{aligned}
$$

Here $\nabla_{\mu}$ is the covariant derivative associated with the metric $g_{\mu \nu}$, the tensor $\perp_{\mu \nu}$ gives the metric on a slice of fixed time while $D^{2}$ is proportional to the Laplacian operator on the same surface, $\lambda$ is the Lagrange multiplier, and the parameters $b_{1}$ and $d_{1}$ with no dimensions and the dimensions of mass square, respectively, are constrained by the astrophysical observations. For the modified dispersion relation (3.1) with $s=2$ and $b_{2}>0$, (3.4) should be replaced with $L_{\text {cor }}=\left(-b_{2} / M^{4}\right)\left(D^{21} \phi\right)\left(D^{22} \phi\right)$, where the operator $D^{2 n}$ is 
defined as $D^{2 n} \equiv D_{\mu_{1}} D^{\mu_{1}} \cdots D_{\mu_{n}} D^{\mu_{n}}$. Then using $z=k|\tau|=k / a h$, (2.26) becomes in the continuum limit $\left(\omega_{l} \rightarrow k\right)$

$$
i \frac{\partial \psi}{\partial t}=\sum_{r k}\left\{-\frac{1}{2} \frac{\partial^{2}}{\partial \phi_{r k}^{2}}+\frac{1}{2}\left[z^{2}\left(1+\sigma^{2} z^{2 s}\right) h^{2}-v^{2} h^{2}\right] \phi_{r k}^{2}\right\} \psi
$$

where $\sigma^{2} \equiv b_{s}(h / M)^{2 s}$, and the ground state wave functional of (3.7) becomes

$$
\psi_{(0)}=\prod_{r k} A_{k(0)}(\tau) \exp \left(-\frac{1}{2} B_{k}(\tau) a^{-1} \phi_{r k}^{2}\right)
$$

where $B_{k}(\tau)$ satisfies

$$
\begin{aligned}
& B_{k}{ }^{2}(\tau)-i\left[B_{k}{ }^{\prime}(\tau)+\frac{B_{k}(\tau)}{\tau}\right]-\left[k^{2}\left(1+\sigma^{2} z^{2 s}\right)-\frac{v^{2}}{\tau^{2}}\right]=0, \\
& B_{k}{ }^{\prime}(\tau) \equiv \frac{d B_{k}(\tau)}{d \tau} \\
& A_{k(0)}(\tau)=\exp \left[-i \frac{1}{2} \int B_{k}(\tau) d \tau+\text { const }\right] .
\end{aligned}
$$

In region I where $k_{\text {phys }} \equiv k / a>M$, that is, $z>M / h$, the dispersion relations can be approximated by $\omega^{2}(k / a) \approx k^{2} \sigma^{2} z^{2 s}$, and (3.9) becomes

$$
B_{k}^{\mathrm{I}^{2}}(\tau)-i\left[B_{k}^{\mathrm{I}^{\prime}}(\tau)+\frac{B_{k}^{\mathrm{I}}(\tau)}{\tau}\right]-\left(k^{2} \sigma^{2} z^{2 s}-\frac{\nu^{2}}{\tau^{2}}\right)=0 .
$$

To obtain the solution of (3.11), we define $B_{k}{ }^{\mathrm{I}}(\tau) \equiv-i f_{k}^{\mathrm{I}^{\prime}} / f_{k}{ }^{I}$, where $f_{k}{ }^{\mathrm{I}^{\prime}}(\tau) \equiv d f_{k}{ }^{\mathrm{I}}(\tau) / d \tau$, and transform (3.11) into

$$
\frac{d^{2} f_{k}{ }^{\mathrm{I}}}{d z^{2}}+\frac{1}{z} \frac{d f_{k}^{\mathrm{I}}}{d z}+\left(\sigma^{2} z^{2 s}-\frac{v^{2}}{z^{2}}\right) f_{k}{ }^{\mathrm{I}}=0
$$

The general solution of (3.12) is

$$
f_{k}^{\mathrm{I}}(z)=C_{1}^{\mathrm{I}}\left(z_{0}\right) H_{\bar{v}}^{(1)}\left(\frac{\sigma}{1+s} z^{1+s}\right)+C_{2}^{\mathrm{I}}\left(z_{0}\right) H_{\bar{v}}^{(1)^{*}}\left(\frac{\sigma}{1+s} z^{1+s}\right)
$$

where the Hankel function is of order $\bar{v}=v / 1+s$ with $v^{2}=9 / 4-\left(m^{2}+\xi R\right) / h^{2}$, and the $k$-dependent constants $C_{1}{ }^{\mathrm{I}}$ and $C_{2}{ }^{\mathrm{I}}$ are to be fixed by choosing suitable initial condition at an arbitrary initial time $\tau_{0}$ for each of the modes and satisfying $\left|C_{1}\right|^{2}-\left|C_{2}\right|^{2}=1$ from the 
Wronskian of $f_{k}{ }^{\mathrm{I}}$ and $f_{k}{ }^{{ }^{*}}$. We can choose $C_{1}{ }^{\mathrm{I}}=1$ and $C_{2}{ }^{\mathrm{I}}=0$ for the initial Bunch-Davies vacuum state and obtain that

$$
B_{k}{ }^{\mathrm{I}}(\tau)=\frac{2 / \pi|\tau|}{\left|H_{\bar{\nu}}{ }^{(1)}\right|^{2}}(1+s)-i \frac{\omega_{l}}{2} \frac{\left(\left|H_{\bar{v}^{(1)}}\right|^{2}\right)^{\prime}}{\left|H_{\bar{v}}{ }^{(1)}\right|^{2}} \sigma z^{s},
$$

where the prime in (3.14) denotes the derivative with respect to $(\sigma /(1+s)) z^{1+s}$. The corresponding wave functional is

$$
\begin{aligned}
\psi_{(0)}{ }^{\mathrm{I}} & =\prod_{r k} A_{k(0)}{ }^{\mathrm{I}}(\tau) \exp \left(-\frac{1}{2} B_{k}^{\mathrm{I}}(\tau) a^{-1}{\phi_{r k}}^{\mathrm{I}^{2}}\right), \\
A_{k(0)}{ }^{\mathrm{I}}(\tau) & =\exp \left[-i \frac{1}{2} \int B_{k}^{\mathrm{I}}(\tau) d \tau+\mathrm{const}\right] .
\end{aligned}
$$

In region II where $k_{\text {phys }} \equiv k / a<M$, that is, $z<M / h$, the dispersion relations recover the standard linear relations $\omega^{2} \cong k^{2}$, and (3.9) becomes

$$
B_{k}{ }^{\mathrm{II}^{2}}(\tau)-i\left[B_{k}{ }^{\mathrm{II}^{\prime}}(\tau)+\frac{B_{k}{ }^{\mathrm{II}}(\tau)}{\tau}\right]-\left(k^{2}-\frac{v^{2}}{\tau^{2}}\right)=0 .
$$

Again, the solution of (3.16) can be obtained by defining $B_{k}{ }^{\mathrm{II}}(\tau) \equiv-i f_{k}{ }^{\mathrm{II}^{\prime}} / f_{k}{ }^{\mathrm{II}}$ and transforming (3.16) into

$$
\frac{d^{2} f_{k}^{\mathrm{II}}}{d z^{2}}+\frac{1}{z} \frac{d f_{k}^{\mathrm{II}}}{d z}+\left(1-\frac{v^{2}}{z^{2}}\right) f_{k}{ }^{\mathrm{II}}=0
$$

The general solution of (3.17) is

$$
f_{k}{ }^{\mathrm{II}}(z)=C_{1}{ }^{\mathrm{II}}\left(z_{0}\right) H_{v}{ }^{(1)}(z)+C_{2}{ }^{\mathrm{II}}\left(z_{0}\right) H_{v}{ }^{(1)^{*}}(z)
$$

where $C_{1}{ }^{\mathrm{II}}$ and $C_{2}{ }^{\mathrm{II}}$ satisfy $\left|C_{1}{ }^{\mathrm{II}}\right|^{2}-\left|C_{2}{ }^{\mathrm{II}}\right|^{2}=1$ from the Wronskian of $f_{k}{ }^{\mathrm{II}}$ and $f_{k}{ }^{\mathrm{II}}$. Therefore, we have

$$
\begin{aligned}
B_{k}{ }^{\mathrm{II}}(\tau)= & \frac{2 / \pi|\tau|}{\left(\left|C_{1}{ }^{\mathrm{II}}\right|^{2}+\left|C_{2}{ }^{\mathrm{II}}\right|^{2}\right)\left|H_{v}{ }^{(1)}\right|^{2}+2 \operatorname{Re}\left[C_{1}{ }^{\mathrm{II}} C_{2}{ }^{\mathrm{II}}\left(H_{v}{ }^{(1)}\right)^{2}\right]} \\
& -i \frac{k}{2} \frac{\left\{\left(\left|C_{1}{ }^{\mathrm{II}}\right|^{2}+\left|C_{2}{ }^{\mathrm{II}}\right|^{2}\right)\left|H_{v}{ }^{(1)}\right|^{2}+2 \operatorname{Re}\left[C_{1}{ }^{\mathrm{II}} C_{2}{ }^{\mathrm{II}}\left(H_{v}{ }^{(1)}\right)^{2}\right]\right\}^{\prime}}{\left(\left|C_{1}{ }^{\mathrm{II}}\right|^{2}+\left|C_{2}{ }^{\mathrm{II}}\right|^{2}\right)\left|H_{v}{ }^{(1)}\right|^{2}+2 \operatorname{Re}\left[C_{1}{ }^{\mathrm{II}} C_{2}{ }^{\mathrm{II}}\left(H_{v}{ }^{(1)}\right)^{2}\right]}
\end{aligned}
$$


where the prime in (3.19) denotes the derivative with respect to $z$. Note that (3.19) can be simply obtained from (2.20) by replacing $\left|H_{v}{ }^{(1)}\right|$ in (2.20) according to [25]

$$
\left|H_{v}^{(1)}\right| \longrightarrow\left\{\left(\left|C_{1}\right|^{2}+\left|C_{2}\right|^{2}\right)\left|H_{v}{ }^{(1)}\right|^{2}+2 \operatorname{Re}\left[C_{1} C_{2}{ }^{*}\left(H_{v}{ }^{(1)}\right)^{2}\right]\right\}^{1 / 2}
$$

The corresponding wave functional is

$$
\begin{gathered}
\psi_{(0)}^{\mathrm{II}}=\prod_{r k} A_{k(0)}{ }^{\mathrm{II}}(\tau) \exp \left(-\frac{1}{2} B_{k}^{\mathrm{II}}(\tau) a^{-1} \phi_{r k}{ }^{\mathrm{II}}\right), \\
A_{k(0)}{ }^{\mathrm{II}}(\tau)=\exp \left[-i \frac{1}{2} \int B_{k}^{\mathrm{II}}(\tau) d \tau+\text { const }\right] .
\end{gathered}
$$

Let $\tau_{c}$ be the time when the modified dispersion relations take the standard linear form. Then $\sigma^{2} z_{c}{ }^{2 s}=1$, where $z_{c}=k\left|\tau_{c}\right|=\left(1 / b_{s}{ }^{1 / 2 s}\right)(M / h) \gg 1$ for $b_{s} \ll 1$. The constants $C_{1}{ }^{\text {II }}$ and $C_{2}{ }^{\mathrm{II}}$ can be obtained by the following matching conditions at $\tau_{c}$ for the two wave functionals (3.15) and (3.21):

$$
\begin{gathered}
\left.\psi_{(0)}{ }^{\mathrm{I}}\right|_{Z_{C}}=\left.\psi_{(0)}{ }^{\mathrm{II}}\right|_{Z_{C}{ }^{\prime}} \\
\left.\frac{d \psi_{(0)}{ }^{\mathrm{I}}}{d z}\right|_{Z_{C}}=\left.\frac{d \psi_{(0)}{ }^{\mathrm{II}}}{d z}\right|_{Z_{C}},
\end{gathered}
$$

which can also be rewritten, respectively, as

$$
\begin{gathered}
\left.\operatorname{Re}\left(B_{k}{ }^{\mathrm{I}}\right)\right|_{Z_{\mathrm{C}}}=\left.\operatorname{Re}\left(B_{k}{ }^{\mathrm{II}}\right)\right|_{Z_{\mathrm{C}}{ }^{\prime}} \\
\left.\frac{d \operatorname{Re}\left(B_{k}^{\mathrm{I}}\right)}{d z}\right|_{Z_{\mathrm{C}}}=\left.\frac{d \operatorname{Re}\left(B_{k}{ }^{\mathrm{II}}\right)}{d z}\right|_{Z_{C}},
\end{gathered}
$$

by using $B_{k}{ }^{\mathrm{I}}=B_{k}{ }^{\mathrm{II}}, \phi_{r k}{ }^{\mathrm{I}}=\phi_{r k}{ }^{\mathrm{II}}, A_{k(0)}{ }^{\mathrm{I}}=A_{k(0)}{ }^{\mathrm{II}}$ when $z=z_{c}$.

To find $C_{1}{ }^{I I}$ and $C_{2}{ }^{I I}$, we focus on the case of massless minimally coupled $(v=3 / 2)$ scalar field in the slowroll inflation and take the CJ type dispersion relation (3.1) with $s=2$ and $b_{2}>0$ which corresponds to the ultraviolet limit of HL gravity. For this case $\bar{v}=1 / 2$, we have $\left|H_{1 / 2}{ }^{(1)}\left((\sigma / 3) z^{3}\right)\right|=\left(6 / \pi \sigma z^{3}\right)^{1 / 2}$ with $\sigma=z_{c}{ }^{-2}$. Then from (3.14), (3.19), and (3.24), we obtain that

$$
1=\left|C_{1}{ }^{\mathrm{II}}\right|^{2}+\left|C_{2}{ }^{\mathrm{II}}\right|^{2}+2\left|C_{1}{ }^{\mathrm{II}}\right|\left|C_{2}{ }^{\mathrm{II}}\right| \cos \left(2 z_{c}-\theta\right)
$$


where we have used $C_{1}{ }^{\mathrm{II}} C_{2}{ }^{\mathrm{I}}{ }^{*}=\left|C_{1}{ }^{\mathrm{II}}\right|\left|C_{2}{ }^{\mathrm{II}}\right| \exp (-\mathrm{i} \theta), \sigma=z_{c}{ }^{-2}$, and $z_{c} \gg 1$. (Note that since $\left|C_{1}{ }^{\mathrm{II}}\right|^{2}-\left|C_{2}{ }^{\mathrm{II}}\right|^{2}=1$ is one real condition on the two complex numbers $C_{1}{ }^{\mathrm{II}}$ and $C_{2}{ }^{\mathrm{II}}$, we can choose $C_{1}{ }^{\mathrm{II}}=\left|C_{1}{ }^{\mathrm{II}}\right|$ and $C_{2}{ }^{\mathrm{II}}=\left|C_{2}{ }^{\mathrm{II}}\right| \exp (i \theta)$, where $\theta$ is the relative phase between $C_{1}{ }^{\mathrm{II}}$ and $C_{2}{ }^{\mathrm{II}}$ and can be considered as a state parameter.) Therefore from (3.26) and $\left|C_{1}{ }^{\mathrm{II}}\right|^{2}-\left|C_{2}{ }^{\mathrm{II}}\right|^{2}=1$ we have

$$
\left|C_{1}{ }^{\mathrm{II}}\right|=\csc \left(2 z_{c}-\theta\right), \quad\left|C_{2}^{\mathrm{II}}\right|=-\cot \left(2 z_{c}-\theta\right),
$$

where $\sin \left(2 z_{c}-\theta\right)>0, \cos \left(2 z_{c}-\theta\right)<0$. Substituting (3.14) and (3.19) into (3.25) and keeping terms up to order $1 / z_{c}$ on the right-hand side of (3.25), we obtain the following:

$$
\frac{2}{z_{c}}=\left|C_{1}{ }^{\mathrm{II}}\right|\left|C_{2}{ }^{\mathrm{II}}\right| \cos \left(2 z_{c}-\theta\right) \frac{8}{z_{c}}+4\left|C_{1}^{\mathrm{II}}\right|\left|C_{2}{ }^{\mathrm{II}}\right| \sin \left(2 z_{c}-\theta\right) .
$$

Using (3.27) in (3.28) gives

$$
\cot \left(2 z_{c}-\theta\right)=-\frac{1}{2 z_{c}} \text { or } \quad \cot \left(2 z_{c}-\theta\right)=-\frac{z_{c}}{2}+\frac{1}{2 z_{c}} .
$$

Here we choose $\cot \left(2 z_{c}-\theta\right)=-1 / 2 z_{c}$, so that $\left|C_{2}{ }^{\mathrm{II}}\right|$ is small for $z_{c} \gg 1$ to avoid an unacceptably large backreaction on the background geometry. Therefore, we have for $z_{c} \gg 1$

$$
\left|C_{2}{ }^{\mathrm{II}}\right| \cong \frac{1}{2 z_{c}}, \quad\left|C_{1}{ }^{\mathrm{II}}\right|=\sqrt{1+\left|C_{2}{ }^{\mathrm{II}}\right|^{2}} \cong 1+\frac{1}{8 z_{c}^{2}} \cong 1
$$

or

$$
\sin \left(2 z_{c}-\theta\right) \cong 1, \quad \cos \left(2 z_{c}-\theta\right) \cong-\frac{1}{2 z_{c}} .
$$

\section{Bohm Quantum Trajectories in the Trans-Planckian Physics}

Note that in defining the pilot-wave scalar field theory in Section 2, we used de Broglie's firstorder dynamics of 1927, which is defined by (2.26) and (2.30). To consider the effect of the trans-Planckian physics, we replace (2.26) with (3.7). In fact, we can also make use of Bohm's second-order dynamics of 1952, which is defined by (3.7) and the following equation in the continuum limit $\left(\omega_{l} \rightarrow k\right)$ :

$$
\frac{d^{2} \phi_{r k}}{d t^{2}}=-\frac{\partial}{\partial \phi_{r k}}(V+Q)
$$

where the classical potential $V$ is given by

$$
V=\sum_{r k} \frac{1}{2}\left[z^{2}\left(1+\sigma^{2} z^{2 s}\right) h^{2}-v^{2} h^{2}\right] \phi_{r k}{ }^{2}
$$


and the so-called "quantum potential" $Q$ is given by

$$
Q=-\sum_{r k} \frac{1}{2\left|\psi_{(0)}\right|} \frac{\partial^{2}\left|\psi_{(0)}\right|}{\partial \phi_{r k}^{2}}
$$

where $\psi_{(0)}$ is given by (3.8)-(3.10), and $\left|\psi_{(0)}\right|$ is given by (2.24) for $n_{r l}=0$. It has been pointed out in previous work [26] that Bohm's second-order dynamics in general leads to more possible quantum trajectories than de Broglie's first-order dynamics does, because Bohm regarded (4.1) as the law of motion, with the de Broglie guidance equation (2.32) added as a constraint on the initial momenta. This distinction between Bohm's second-order dynamics and de Broglie's first-order dynamics was also emphasized recently by Valentini [29].

In region I where $\omega^{2}(k / a) \approx k^{2} \sigma^{2} z^{2 s}$ and $z>M / h$, the classical potential $V$ in (4.2) becomes

$$
V=\sum_{r k} \frac{1}{2}\left(\sigma^{2} z^{2+2 s} h^{2}-v^{2} h^{2}\right) \phi_{r k}{ }^{2}
$$

and from (4.3), (3.8), (3.10), and (3.14), the quantum potential $Q$ becomes

$$
Q=\sum_{r k}\left(-\frac{1}{2} \frac{(2 h / \pi)^{2}}{\left|H_{\bar{v}^{(1)}}\right|^{4}}(1+s)^{2} \phi_{r k}{ }^{2}+\frac{1}{2} \frac{2 h / \pi}{\left|H_{\bar{\nu}}(1)\right|^{2}}(1+s)\right)
$$

Substituting (4.4) and (4.5) into (4.1) and using $d \tau=d t / a$ and $z=k|\tau|=k / a h$ gives

$$
z^{2} \frac{d^{2} \phi_{r k}^{\mathrm{I}}}{d z^{2}}+z \frac{d \phi_{r k}^{\mathrm{I}}}{d z}+\left[\sigma^{2} z^{2+2 s}-v^{2}-\frac{4}{\pi^{2}}\left|H_{\bar{v}^{(1)}}\right|^{-4}(1+2 s)^{2}\right] \phi_{r k}^{\mathrm{I}}=0
$$

On the other hand, in region II where $\omega^{2}(k / a) \approx k^{2}$ and $z<M / h$, the classical potential $V$ in (4.1) and the corresponding quantum potential $Q$ become, respectively,

$$
\begin{aligned}
& V=\sum_{r k} \frac{1}{2}\left(z^{2} h^{2}-v^{2} h^{2}\right) \phi_{r k}{ }^{\mathrm{II}^{2}} \\
& Q=\sum_{r k}\left(-\frac{1}{2} \frac{(2 h / \pi)^{2}}{\left|H_{v}{ }^{(1)}\right|_{\mathrm{md}}^{4}} \phi_{r k}{ }^{\mathrm{II}^{2}}+\frac{1}{2} \frac{2 h / \pi}{\left|H_{v}{ }^{(1)}\right|_{\mathrm{md}}{ }^{2}}\right)
\end{aligned}
$$

where $\left|H_{v}{ }^{(1)}\right|_{\text {md }}$ means $\left|H_{v}{ }^{(1)}\right|$ modified according to (3.20). Then the quantum trajectory $\phi_{r k}{ }^{\text {II }}$ satisfies

$$
z^{2} \frac{d^{2} \phi_{r k}^{\mathrm{II}}}{d z^{2}}+z \frac{d \phi_{r k}^{\mathrm{II}}}{d z}+\left[z^{2}-v^{2}-\frac{4}{\pi^{2}}\left|H_{v}^{(1)}\right|_{\mathrm{md}}^{-4}\right] \phi_{r k}^{\mathrm{II}}=0 .
$$


In particular, for the case of massless minimally coupled $(v=3 / 2)$ scalar field and Corley-Jacobson dispersion relation (3.1) with $s=2$, (4.6) becomes

$$
z^{2} \frac{d^{2} \phi_{r k}^{\mathrm{I}}}{d z^{2}}+z \frac{d \phi_{\mathrm{rk}}^{\mathrm{I}}}{d z}-\frac{9}{4} \phi_{r k}^{\mathrm{I}}=0
$$

because in this case the first term in the square brackets of (4.6) is exactly cancelled by the last term. The general solution of (4.9) is

$$
\phi_{r k}^{\mathrm{I}}(z)=\overline{\mathrm{C}}_{1}^{\mathrm{I}} z^{-3 / 2}+\overline{\mathrm{C}}_{2}^{\mathrm{I}} z^{3 / 2}
$$

where $\bar{C}_{1}{ }^{\mathrm{I}}$ and $\bar{C}_{2}{ }^{\mathrm{I}}$ are constants to be fixed by choosing suitable initial conditions at an arbitrary initial time $\tau_{0}$ for the quantum trajectory $\phi_{r k}{ }^{\mathrm{I}}$. Here we can choose $\bar{C}_{1}^{\mathrm{I}} \neq 0$ and $\bar{C}_{2}{ }^{\mathrm{I}}=0$ so that the first term $\bar{C}_{1}{ }^{\mathrm{I}} z^{-3 / 2}$ of $\phi_{r k}{ }^{\mathrm{I}}$ corresponds to $(2.33)$ with $\left|H_{1 / 2}{ }^{(1)}\left((\sigma / 3) z^{3}\right)\right|=$ $\left(6 / \pi \sigma z^{3}\right)^{1 / 2}$. On the other hand, in the region II $\left|H_{3 / 2}{ }^{(1)}\right|_{\text {md }}$ becomes

$$
\begin{aligned}
\left|H_{3 / 2}{ }^{(1)}\right|_{\mathrm{md}}= & \left|H_{3 / 2}{ }^{(1)}\right|\left\{\left|C_{1}{ }_{1}^{\mathrm{II}}\right|^{2}+\left|C_{2}{ }^{\mathrm{II}}\right|^{2}+2\left|C_{1}{ }^{\mathrm{II}}\right|\left|C_{2}{ }^{\mathrm{II}}\right|\right. \\
& \left.\times\left[\cos (2 z-\theta) \frac{z^{2}-1}{z^{2}+1}-\sin (2 z-\theta) \frac{2 z}{1+z^{2}}\right]\right\} .
\end{aligned}
$$

For $z \rightarrow z_{c} \gg 1$ (4.11) reduces to $\left|H_{3 / 2}{ }^{(1)}\right|$ by using (3.26), and the quantum trajectory $\phi_{r k}{ }^{\text {II }}$ satisfies

$$
z^{2} \frac{d^{2} \phi_{r k}{ }^{\mathrm{II}}}{d z^{2}}+z \frac{d \phi_{r k}^{\mathrm{II}}}{d z}+\left[z^{2}-\frac{9}{4}-\frac{4}{\pi^{2}}\left|H_{3 / 2}{ }^{(1)}\right|^{-4}\right] \phi_{r k}^{\mathrm{II}}=0 .
$$

The most general asymptotic series solution of (4.12) is [26]

$$
\phi_{r k}{ }^{\mathrm{II}}(z)=\overline{\mathrm{C}}_{1}{ }^{\mathrm{II}} z^{-1 / 2}\left(1+\frac{1}{2} z^{-2}-\frac{1}{8} z^{-4}+\cdots\right)+\overline{\mathrm{C}}_{2}{ }^{\mathrm{II}} z^{1 / 2}\left(1+\frac{3}{2} z^{-2}+\frac{1}{24} z^{-4}+\cdots\right) .
$$

For $z \rightarrow z_{c} \gg 1$, the solution (4.13) reduces to

$$
\phi_{r k}{ }^{\mathrm{II}}(z) \approx \overline{\mathrm{C}}_{1}^{\mathrm{II}} z^{-1 / 2}+\overline{\mathrm{C}}_{2}^{\mathrm{II}} z^{1 / 2} .
$$


Substituting (4.10) and (4.14) into the following matching conditions at $z_{c}$ for the two quantum trajectories $\phi_{r k}{ }^{\mathrm{I}}$ and $\phi_{r k}{ }^{\mathrm{II}}$ :

$$
\begin{gathered}
\left.\phi_{r k}{ }^{\mathrm{I}}\right|_{Z_{C}}=\left.\phi_{r k}{ }^{\mathrm{II}}\right|_{Z_{C}{ }^{\prime}} \\
\left.\frac{d \phi_{r k}{ }^{\mathrm{I}}}{d z}\right|_{Z_{C}}=\left.\frac{d \phi_{r k}{ }^{\mathrm{II}}}{d z}\right|_{Z_{C}},
\end{gathered}
$$

we obtain

$$
\bar{C}_{1}{ }^{I I}=2 \bar{C}_{1}{ }^{I} \frac{1}{z_{c}}, \quad \bar{C}_{2}{ }^{I I}=-\bar{C}_{1}{ }^{I} \frac{1}{z_{c}^{2}} .
$$

From (4.14) and (4.16) we see that as $z$ decreases from $z_{c}$ to $1, \bar{C}_{1}{ }^{\text {II }} z^{-1 / 2}$ becomes the dominant term, that is,

$$
\phi_{r k}^{\mathrm{II}}(z) \approx \overline{\mathrm{C}}_{1}^{\mathrm{II}} z^{-1 / 2}
$$

On the other hand, for $z \ll 1$ (well after horizon exit), (4.11) also reduces to $\left|H_{3 / 2}{ }^{(1)}\right|$ by using (3.30) and $z_{c} \gg 1$, and the most general power series solution of (4.12) is [26]

$$
\begin{aligned}
\phi_{r k}^{\mathrm{II}}(z)= & \widehat{C}_{1}^{\mathrm{II}} z^{-3 / 2}\left(1+\frac{1}{2} z^{2}-\frac{1}{8} z^{4}+\cdots\right)+\widehat{C}_{2}^{\mathrm{II}} z^{3 / 2} \\
& \times\left(1-\frac{1}{10} z^{2}+\frac{1}{280} z^{4}+\cdots\right) .
\end{aligned}
$$

Note that for $z \ll 1$, the last term in the square brackets of (4.12) is negligible when compared with the first term, and (4.12) becomes a Bessel equation with the solution

$$
\phi_{r k}(z)=C N_{3 / 2}(z)
$$

where $N_{3 / 2}(z)$ is the Neumann function which has the following expression:

$$
N_{3 / 2}(z) \longrightarrow-\frac{\Gamma(3 / 2)}{\pi}\left(\frac{2}{z}\right)^{3 / 2}, \quad z \ll 1
$$

and $C$ is a constant. Note also that, for $z \ll 1$, (4.19) is equivalent to (2.33). Therefore, for $z \ll 1$, the solution (4.18) reduces to

$$
\phi_{r k}^{\mathrm{II}}(z) \approx \widehat{C}_{1}^{\mathrm{II}} z^{-3 / 2}
$$


which corresponds to (4.19) and (4.20). Requiring $\phi_{r k}{ }^{\mathrm{II}}$ to be continuous at $z=1$ (for the justification of this patching condition, see the discussion (iv) in Section 5), we have from (4.17), (4.21), and (4.16)

$$
\widehat{C}_{1}^{\mathrm{II}}=\overline{\mathrm{C}}_{1}^{\mathrm{II}}=\frac{2}{z_{c}} \overline{\mathrm{C}}_{1}^{\mathrm{I}}
$$

Since in the case of $d=3, \phi_{r k}$ contains a factor $a^{3 / 2}$ which is proportional to $z^{-3 / 2}$, we can define a new field variable $u_{r k} \equiv a^{-3 / 2} \phi_{r k}$ and use $a=(k / h) z^{-1}$ to rewrite (4.10) and (4.21) as

$$
u_{r k}^{\mathrm{I}}=\left(\frac{k}{h}\right)^{-3 / 2} \bar{C}_{1}^{\mathrm{I}}, \quad u_{r k}^{\mathrm{II}}=\frac{2}{z_{c}}\left(\frac{k}{h}\right)^{-3 / 2} \bar{C}_{1}^{\mathrm{I}} .
$$

Thus, we see from (4.23) that for fixed $k$ and $z_{c} \gg 1$, as $z$ decreases from $z \gg 1$ to $z \ll 1$, the scalar field evolves from one constant to another much smaller constant; that is, there is a transition in the time evolution of the quantum trajectory of the scalar field.

\section{Conclusion and Discussion}

In this paper we have considered a generically coupled free real scalar field in de Sitter space in the lattice Schrödinger picture within the framework of the pilot-wave theory of de Broglie and Bohm. In particular, we have investigated the possible effects of the trans-Planckian physics on the quantum trajectories of the vacuum state of scalar field.

For the massless minimally coupled scalar field and the Corley-Jacobson type dispersion relation with sextic correction to the standard-squared linear relation, we have found that as $z$ decreases from $z \gg 1$ (well before horizon exit) to $z \ll 1$ (well after horizon exit), there is a transition in the time evolution of the quantum trajectory of the scalar field.

For the massive nonminimally coupled scalar field $\left(v^{2}=9 / 4-\left(m^{2}+\xi R\right) / h^{2}\right)$ which is relevant to the nonminimally coupled chaotic inflation with quadratic potential $V=m^{2} \phi^{2} / 2$, the coupling to the curvature $\xi R \phi^{2} / 2$ leads to the additional mass-squared $m^{2}=12 \xi h^{2}$ in the case of $d=3$. Notice that $m^{2} \ll h^{2}$ and $|\xi| \ll 1$ are required for slowroll [30,31]. Thus, in general, we have $v \cong 3 / 2$, and expect that for the massive nonminimally coupled scalar field and the Corley-Jacobson-type dispersion relation with sextic correction, the evolution of the quantum trajectory of the scalar field also exhibits similar transitional behavior from $z \gg 1$ to $z \ll 1$.

Finally we conclude this paper with the following discussions.

(i) Since a constant scalar field is similar to a cosmological constant $\Lambda$, the transition could be interpreted as a transition of the Universe from a large $\Lambda$ to a small $\Lambda$, thus providing a possible mechanism to solve the riddle of the smallness of $\Lambda$ in the framework of the Bohmian approach to quantum theory of inflationary cosmology. Note that the vacuum energy density due to quantum states with $k<k_{\max }$ is $\rho_{\text {vac }} \cong k_{\max }{ }^{4} \sim M_{\mathrm{Pl}}{ }^{4}$, and $\rho_{\text {vac }}=$ $M_{\mathrm{Pl}}^{2} \Lambda / 8 \pi$ (here $M_{\mathrm{Pl}}=1.22 \times 10^{19} \mathrm{Gev}$ is the Planck mass). For the simplest potential driving inflation $V(\phi)=m^{2} \phi^{2} / 2$, from (4.23) and $\rho_{\mathrm{vac}}=V(\phi=$ const $)$, we expect that as $z$ decreases from $z_{c}$ to 0 , the cosmological constant $\Lambda$ decreases from $8 \pi M_{\mathrm{Pl}}^{2}$ to $16 \pi \mathrm{m}^{2} z_{c}{ }^{-2}$. Here, we have $z_{c}=k\left|\tau_{c}\right|=\left(1 / b_{2}{ }^{1 / 4}\right)\left(M_{\mathrm{Pl}} / h\right), M_{\mathrm{Pl}}$ is the Planck cutoff scale, $h \sim 10^{15} \mathrm{Gev}$ is the Hubble constant during inflation, and $m=1.8 \times 10^{13} \mathrm{Gev}$ which can be obtained from COBE normalization (see [1], page 212). Since current observations indicate that 
$\rho_{\text {vac }}{ }^{0} \sim 10^{-47} \mathrm{Gev}^{4}$ and $\Lambda^{0} \sim 10^{-83} \mathrm{Gev}^{2}$, we find that the value of the parameter $b_{2}$ is very small, $b_{2} \sim 10^{-206}$. Therefore the smallness of the cosmological constant is intimately connected with the infinitesimal violation of Lorentz invariance at the level of sextic correction to the standard squared linear dispersion relation.

Since $z_{c} \gg 1$, the transitional time is in the very early stage of inflation. We note that the quantum potential $Q$ plays an important role in the evolution of the quantum trajectory of scalar field. From (4.1) and (4.12), we also see that for $z \gg 1$ and the mode $\phi_{r k}$ II the quantum force $\left(4 h^{2} / \pi^{2}\right)\left|H_{3 / 2}{ }^{(1)}\right|^{-4} \phi_{r k}$ II arising from the quantum potential $Q$ through $-\partial Q / \partial \phi_{r k}^{\text {II }}$ approximately cancels the classical force $-\left(z^{2} h^{2}-9 / 4 h^{2}\right) \phi_{r k}{ }^{\text {II }}$ arising from the classical potential $V$ through $-\partial V / \partial \phi_{r k}{ }^{\text {II }}$, while for $z \ll 1$ the quantum force becomes negligible with respect to the classical force. Therefore, as $z$ decreases from $z_{c}$ to 0 , there is a quantum-to-classical transition. In this regard, the result is the same as that of the recent relevant work in which the quantum-to-classical transition of primordial cosmological perturbations is obtained in the context of the de Broglie-Bohm theory [32].

(ii) In a Lorentz noninvariant theory with the action (3.2), the modified dispersion relation (3.1) breaks the local Lorentz invariance explicitly while it preserves rotational and translational invariance. The phenomenological bounds on the parameters of the theory come from the observations of ultra-high-energy cosmic rays [33]. Using effective field theory with higher-dimensional Lorentz violating operators, which results in the modified field theory with a dispersion relation, it was shown that for various standard model particles the numerical value of the bound on the parameter $b_{1}$ in (3.1) is $b_{1}<5 \times 10^{-5}$ with the Planck cut-off scale $M \cong M_{\mathrm{Pl}}$. On the other hand, the bound on the parameter $b_{2}$ in (3.1) is currently not available observationally, but its numerical value of the bound is expected to be so small as we have shown in the previous discussion when the cut-off scale is near to the Planck scale.

(iii) In the last paragraph of Section 2, we note that the quantum trajectory (2.33) is independent of the quantum number $n_{r l}$. This is due to the fact that the Hermite polynomials in energy eigenstates (2.19) or (2.23) are real. If we consider the state which is some superposition of energy eigenstates such as a wave packet or squeezed state, then we would obtain trajectories that generally depend on the quantum number $n_{r l}$. Moreover, note that in general the trajectories for each mode are not independent of each other. However, since we are dealing with particularly simple state which factories, these issues do not arise here.

(iv) Regarding the patching condition after (4.21), note that, in the region II, $\left|H_{3 / 2}{ }^{(1)}\right|_{\text {md }}$ in (4.11) reduces to $\left|H_{3 / 2}{ }^{(1)}\right|$ as $z$ decreases continuously from $z_{c}$ to 0 (not only for $z \gg 1$ or $z \ll 1$ or $z \cong 1$ ) due to (3.30) and $z_{c} \gg 1$. Therefore, the quantum trajectory $\phi_{r k}$ II $(z)$ satisfies (4.12)

$$
z^{2} \frac{d^{2} \phi_{r k}{ }^{\mathrm{II}}}{d z^{2}}+z \frac{d \phi_{r k}^{\mathrm{II}}}{d z}+\left[z^{2}-\frac{9}{4}-\frac{4}{\pi^{2}}\left|H_{3 / 2}{ }^{(1)}\right|^{-4}\right] \phi_{r k}^{\mathrm{II}}=0
$$

where $\left|H_{3 / 2}{ }^{(1)}(z)\right|=z^{-3 / 2} \sqrt{1+z^{2}}$ for $0 \leq z \leq z_{c}$. Note also that the solution of (2.32). 
$\phi_{r k}(z)=C\left|H_{3 / 2}{ }^{(1)}(z)\right|$ is also one solution of (4.12), which is just the same as that appearing in (4.13) and (4.18). This is because the first asymptotic series solution of (4.13) can be rewritten as

$$
\bar{C}_{1}{ }^{\mathrm{II}} z^{-1 / 2}\left(1+\frac{1}{2} z^{-2}-\frac{1}{8} z^{-4}+\cdots\right)=\overline{\mathrm{C}}_{1}^{\mathrm{II}} z^{-1 / 2} \sqrt{1+\left(\frac{1}{z}\right)^{2}}=\bar{C}_{1}{ }^{\mathrm{II}} z^{-3 / 2} \sqrt{1+z^{2}}
$$

while the first power series solution of (4.18) can be rewritten as

$$
\widehat{C}_{1}^{\mathrm{II}} z^{-3 / 2}\left(1+\frac{1}{2} z^{2}-\frac{1}{8} z^{4}+\cdots\right)=\widehat{C}_{1}^{\mathrm{II}} z^{-3 / 2} \sqrt{1+z^{2}}
$$

Therefore, we have $\widehat{C}_{1}^{\mathrm{II}}=\overline{\mathrm{C}}_{1}^{\mathrm{II}}$.

(v) The pilot-wave model treated in this paper is intrinsically nonlocal because of Bell's theorem and not Lorentz invariant because of the formulation with respect to a preferred frame of reference. However, in quantum equilibrium, the pilot-wave models will reproduce the standard quantum theoretical predictions. In fact, there are some attempts to formulate Lorentz covariant pilot-wave models [34].

\section{Acknowledgments}

The author would like to thank M.-J. Wang for fruitful discussions on trans-Planckian physics, and his colleagues at Ming Chi University of Technology for useful suggestions. The author also would like to thank the anonymous referees for helpful comments.

\section{References}

[1] A. R. Liddle and D. H. Lyth, Cosmological Inflation and Large-Scale Structure, Cambridge University Press, Cambridge, UK, 2000.

[2] T. S. Bunch and P. C. W. Davies, "Quantum field theory in de Sitter space: renormalization by point splitting," Proceedings of the Royal Society A, vol. 360, p. 117, 1978.

[3] N. D. Birrell and P. C. W. Davies, Quantum Fields in Curved Space, Cambridge University Press, Cambridge, UK, 1982.

[4] U. H. Danielsson, "Note on inflation and trans-Planckian physics," Physical Review D, vol. 66, no. 2, Article ID 023511, 2002.

[5] C. Armendáriz-Picón and E. A. Lim, "Vacuum choices and the predictions of inflation," Journal of Cosmology and Astroparticle Physics, vol. 2003, no. 12, article 006, pp. 75-91, 2003.

[6] A. Perez, H. Sahlmann, and D. Sudarsky, "On the quantum origin of the seeds of cosmic structure," Classical and Quantum Gravity, vol. 23, no. 7, pp. 2317-2354, 2006.

[7] A. Valentini, "Signal-locality, uncertainty, and the subquantum H-theorem. I," Physics Letters A, vol. 156, no. 1-2, pp. 5-11, 1991.

[8] A. Valentini, "Signal-locality, uncertainty, and the subquantum H-theorem. II," Physics Letters A, vol. 158, no. 1-2, pp. 1-8, 1991.

[9] L. de Broglie, "Sur la possibilité de relier les phénomènes d'interférences et de diffraction à la théorie des quanta de lumière," Comptes Rendus de l'Académie des Sciences, vol. 183, p. 447, 1926.

[10] L. de Broglie, "La structure atomique de la matière et du rayonnement et la mécanique ondulatoire," Comptes Rendus de l'Académie des Sciences, vol. 184, p. 273, 1927.

[11] L. de Broglie, "Sur le rôle des ondes continues en mécanique ondulatoire," Comptes Rendus de l'Académie des Sciences, vol. 185, p. 380, 1927. 
[12] D. Bohm, "A suggested interpretation of the quantum theory in terms of "hidden" variables. I," Physical Review, vol. 85, no. 2, pp. 166-179, 1952.

[13] D. Bohm, "A suggested interpretation of the quantum theory in terms of "hidden" variables. II," Physical Review, vol. 85, no. 2, pp. 180-193, 1952.

[14] P. Pearle and A. Valentini, "Quantum mechanics: generalizations," in Encyclopaedia of Mathematical Physics, J.-P. Françoise, G. Naber, and T. S. Tsun, Eds., Elsevier, 2006.

[15] A. Valentini, "Astrophysical and cosmological tests of quantum theory," Journal of Physics A, vol. 40, no. 12, pp. 3285-3303, 2007.

[16] A. Valentini, "Inflationary cosmology as a probe of primordial quantum mechanics," Physical Review D, vol. 82, no. 6, Article ID 063513, 2010.

[17] J. Martin and R. H. Brandenberger, "Trans-planckian problem of inflationary cosmology," Physical Review D, vol. 63, no. 12, Article ID 123501, 16 pages, 2001.

[18] R. H. Brandenberger and J. Martin, "The robustness of inflation to changes in super-Planck-scale physics," Modern Physics Letters A, vol. 16, no. 15, pp. 999-1006, 2001.

[19] J. Martin and R. H. Brandenberger, "Corley-Jacobson dispersion relation and trans-Planckian inflation," Physical Review D, vol. 65, no. 10, Article ID 103514, 2002.

[20] P. Hořava, "Quantum gravity at a Lifshitz point," Physical Review D, vol. 79, no. 8, Article ID 084008, 2009.

[21] P. Hořava, "Spectral dimension of the universe in quantum gravity at a Lifshitz point," Physical Review Letters, vol. 102, no. 16, 2009.

[22] S. Koh, "Relic gravitational wave spectrum, the trans-Planckian physics and Hořava-Lifshitz gravity," Classical and Quantum Gravity, vol. 27, no. 22, 2010.

[23] J.-J. Huang and M.-J. Wang, “Green's functions of scalar field in de Sitter space: discrete lattice formalism.-I," Il Nuovo Cimento A, vol. 100, no. 5, pp. 723-734, 1988.

[24] J.-J. Huang, “Excited states of de Sitter space scalar fields: lattice Schrödinger picture," Modern Physics Letters A, vol. 21, no. 22, pp. 1717-1725, 2006.

[25] J.-J. Huang, "Inflation and squeezed states: lattice Schrödinger picture," Modern Physics Letters A, vol. 24, no. 7, pp. 497-508, 2009.

[26] J.-J. Huang, "Pilot-wave scalar field theory in de Sitter space: lattice Schrödinger picture," Modern Physics Letters A, vol. 25, no. 1, pp. 1-13, 2010.

[27] T. Jacobson and D. Mattingly, "Generally covariant model of a scalar field with high frequency dispersion and the cosmological horizon problem," Physical Review D, vol. 63, no. 4, Article ID 041502, 2001.

[28] M. Lemoine, M. Lubo, J. Martin, and J. P. Uzan, "Stress-energy tensor for trans-Planckian cosmology," Physical Review D, vol. 65, no. 2, Article ID 023510, 2002.

[29] A. Valentini, "De Broglie-Bohm pilot-wave theory: many worlds interpretation," in Many Worlds? Everett, Quantum Theory, and Reality, S. Saunders, J. Barrett, A. Kent, and D. Wallace, Eds., pp. 476509, Oxford University Press, Oxford, UK, 2010.

[30] T. Chiba and M. Yamaguchi, "Extended slow-roll conditions and rapid-roll conditions," Journal of Cosmology and Astroparticle Physics, vol. 2008, no. 10, article 021, 2008.

[31] A. Linde, M. Noorbala, and A. Westphal, "Observational consequences of chaotic inflation with nonminimal coupling to gravity," Journal of Cosmology and Astroparticle Physics, vol. 2011, no. 3, article 013, 2011.

[32] N. Pinto-Neto, G. Santos, and W. Struyve, "The quantum-to-classical transition of primordial cosmological perturbations," General Relativity and Quantum Cosmology. In press. http:// arxiv.org/abs/1110.1339.

[33] O. Gagnon and G. D. Moore, "Limits on Lorentz violation from the highest energy cosmic rays," Physical Review D, vol. 70, no. 6, Article ID 065002, 2004.

[34] R. Tumulka, "The "unromantic pictures" of quantum theory," Journal of Physics A, vol. 40, no. 12, pp. 3245-3273, 2007. 



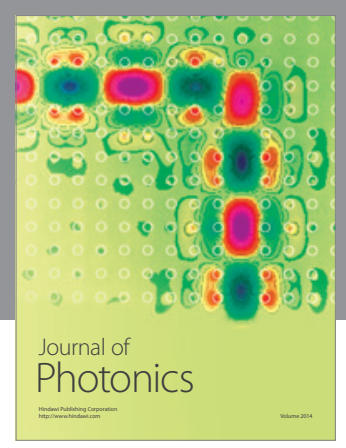

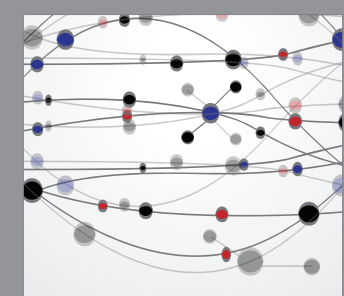

The Scientific World Journal
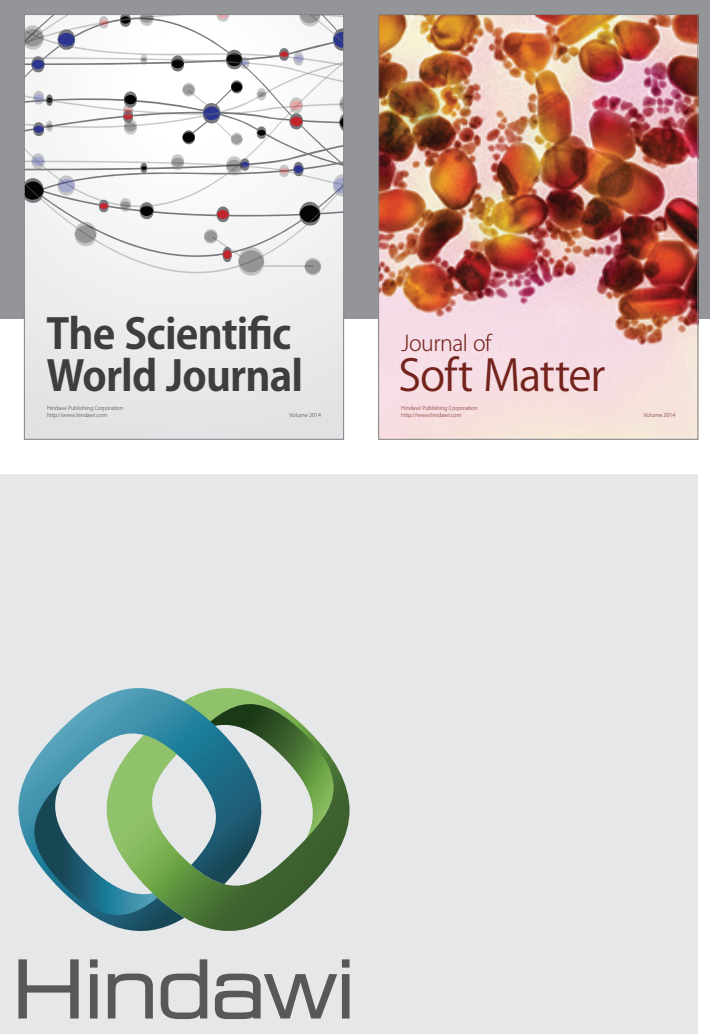

Submit your manuscripts at

http://www.hindawi.com

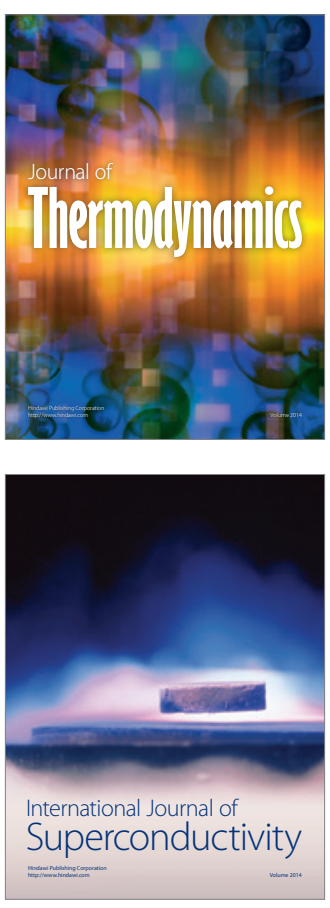
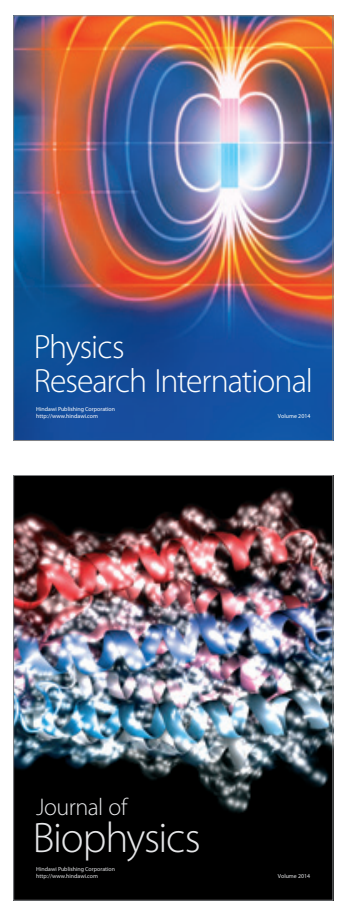
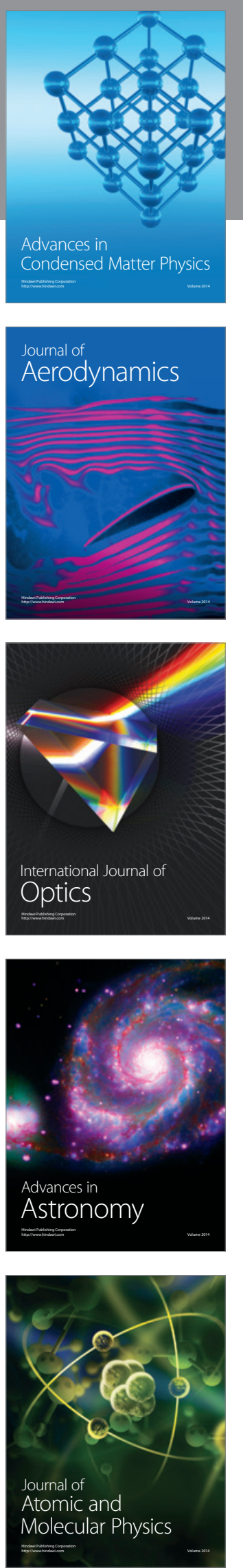